\title{
Towards a Socially-Aware Management of New Overlay Application Traffic Combined with Energy Efficiency in the Internet (SmartenIT)*
}

\author{
Burkhard Stiller ${ }^{1}$, David Hausheer ${ }^{2}$, and Tobias Hoßfeld ${ }^{3}$ \\ ${ }^{1}$ University of Zürich, Department of Informatics, Communication Systems Group, \\ Switzerland \\ ${ }^{2}$ P2P Systems Engineering, TU Darmstadt, Germany \\ ${ }^{3}$ University of Würzburg, Institute of Computer Science, Würzburg, Germany \\ stiller@ifi.uzh.ch, hausheer@ps.tu-darmstadt.de, \\ hossfeldeinformatik. uni-wuerzburg.de
}

\begin{abstract}
The Internet has seen a strong move to support overlay applications, which demand a coherent and integrated control in underlying heterogeneous networks in a scalable, resilient, and energy-efficient manner. A tighter integration of network management and overlay service functionality can lead to crosslayer optimization of operations and management, which is a promising approach as it offers a large business potential in operational perspectives for all players involved. Therefore, the objective of this paper is to present SmartenIT (Socially-aware Management of New Overlay Application Traffic combined with Energy Efficiency in the Internet), which targets at an incentivecompatible cross-layer network management for providers of overlay-based application (e.g., cloud applications, content delivery, and social networks), network providers, and end-users. The goal is to ensure a QoE-awareness, by addressing accordingly load and traffic patterns or special application requirements, and exploiting at the same time social awareness (in terms of user relations and interests). Moreover, energy efficiency with respect to both end-user devices and underlying networking infrastructure is tackled to ensure an operationally efficient management. Incentive-compatible network management mechanisms for improving metrics on an inter-domain basis for ISPs serve as the major mechanism to deal with and investigate real-life scenarios.
\end{abstract}

Keywords: Economic traffic management, application-layer traffic optimization, inter-cloud communications, social networks, QoE, energy efficiency.

\section{Introduction}

There are important new applications influencing volume and patterns of Internet traffic and the satisfaction of users [1]. These include cloud computing and applications served, thereby social networks. Additionally, the Future Internet's entertainment use will generate more traffic to come [2]. Finally, the mobile services and respective wireless access network demand is increasing, too [3], resulting in very

\footnotetext{
* Invited Paper.
}

A. Galis and A. Gavras (Eds.): FIA 2013, LNCS 7858, pp. 3-15, 2013.

(C) The Author(s). This article is published with open access at link.springer.com 
different communication path quality levels and management tasks. In turn, traffic from such overlay applications on a very dramatically increasing number of devices and end-points (wired and mobile) is continuously exploding [4]. Respective network management and operation frameworks are missing today for heterogeneous technologies and modern applications. New management mechanisms should support effectively scale, agility, stable Quality-of-Experience (QoE), and flexibility, in an integrated set of network and overlay service functionality. This calls for a new traffic management approach, that derives three major pillars of the new SmartenIT project:

- Handling the explosion of traffic of overlay and social network service functionality and respective end-points in the wired and wireless domain, cross-cutting multiple operators' domains in support of combined intra-domain cloud services;

- Proposing an agile network management and operation framework for this case, while addressing a better control of heterogeneous networks and their network functionality and integrating overlay service functionality in support of stable QoE;

- Evaluating the new approach by employing highly distributed mechanisms, optimized control, and energy efficiency aspects.

Under this perspective the remainder of this overview tackles in Section 2 the details of current Internet trends. The challenges emerging from those developments are clarified in Section 3. To enable a viable problem solving, use cases and solution approaches are discussed in Section 4, which lead to the basic requirements design of the systems architecture for SmartenIT in Section 5. Finally, Section 6 draws preliminary conclusions of those plans and next steps to come.

\section{Current Trends in the Internet}

The introduction of cloud-based services, cloud technologies, and respective stakeholders in a commercialized internetworking world has changed the type and style of traffic generated for and transported within the Internet. This basic change is complemented by well understood effects of overlay services and applications on the network [5]. However, new trends in terms of social network-generated traffic (putting up demands on Internet Service Providers, ISP) as well as energy efficiency requirements (making updates of ISP-internal technology necessary) do affect the transport of ISP traffic in an unknown manner. Thus, depending on certain criteria of optimization (such as QoE, energy efficiency, and traffic profiles) these trends have to be investigated, especially under the new problem of additional stakeholders in the traditional value chain, addressing additionally Cloud Providers (CP), Social Network Providers (SNP), energy-efficient technologies, and constraints.

Overlay Applications. Overlay applications interconnect computers storing and circulating information among each other with no awareness of the topology and state of the underlying transport network infrastructure; some computers also host computational resources to run applications remotely on virtual platforms. Popular overlay applications include, e.g., Content Delivery (CD), Peer-to-peer (P2P), and social 
networking. Interconnected computers can be personal hosts or end-systems in the user's network edge.

A characteristic of overlay applications is that application endpoints are agnostic of the underlying topology, state, and policy and typically have no detail of where computers they trade content and resources with are physically located. This causes a waste of resources, energy, and revenue for network providers, while degrading the users' QoE and eventually impacting overlay application network providers. This issue is being addressed by Application Providers (AP), who off-load storage of servers and end-systems as well as a user equipment computational burden by moving involved resources into two directions:

- Content Delivery Networks (CDN) move content from origin servers to the end user by disseminating popular contents in caching servers deployed throughout the Internet, shortening the transport path to users and minimizing congestion and server load. Content is retrieved through request routing techniques, such as DNS and HTTP redirect. Likewise, when content is moved across caching servers to fit the social demand, careful decisions need be taken on which content to move, where and when. Akamai (a pioneer in CDNs) operates more than 100,000 caching servers, trying to span the Internet. Other CDNs attempt to CD and provide ISPs with their technology, enabling them to run their own CDN to peer with ISPs having the same CDN technology upon optimizing their local traffic offer, such as Velocix serving Verizon or New Zealand's Orcon. Finally, CDNs are heavily used by social networking providers such as Facebook.

- CPs and APs make movements from the user to the network, by delegating storage and computation burden to the network, which is more valuable for devices with limited resources and access, such as mobile terminals or those located in regions with sparse infrastructure. Clouded applications run over virtual platforms, where actual resources are distributed over several physical machines.

Both CDNs and cloud networks use resources physically located in Data Centers (DC), the meeting point of content and resource movements from and to users. DCs host resources to jointly offer cloud computing, Web services, and CD, e.g., Amazon's Cloudfront CDN. These applications include a selection of physical server/machine(s) to (1) store or get requested content from in a CDN and (2) provide resources requested for a "clouded" application.

On the one hand, CDN or CPs have a too coarse insight into the underlying transport infrastructure as they usually span across several ISP domains. CDNs use request routing to retrieve content and choose the most suitable content location, but lack the visibility on the underlying transport topology that would enable to minimize the routing cost and provide their customers with the best possible QoE. On the other hand, ISPs want to minimize costly transit traffic caused by inter-domain content movements. ISPs running their CD service often need to get the content from other CDNs and to this end need to exchange information to retrieve the "best" location.

Despite numerous proprietary technologies there is no standardized way to distribute infrastructure information to overlay applications or support information exchange between CDNs. One way to do the latter is the use of protocols being 
specified by the IETF CDNi (CDN interconnection) WG [6]. The IETF ALTO clientserver protocol addresses underlay network agnosticity by providing abstracted routing cost information that can be used by the request routing process and any location selection process to comply with the policy of the impacted ISPs. Likewise, orchestration systems for clouded applications, when selecting the location of needed physical resources, have the same need as CDNs selecting locations in different domains. The use of ALTO in CDNi to optimize request routing decisions is being promoted in ALTO and CDNi. The ALTO protocol is seen as "layer cooperative" in that it involves information being provided by one layer to an upper layer through an explicit protocol. Abstraction is definitely the use of an incentive to the owner of the reported network. Further work is needed to (1) extend the ALTO services and information to support CDN and Data Centers based overlay applications, (2) specify ALTO information needed for CDNi, and (3) specify ALTO protocol extensions to ensure provider confidentiality.

The SmartenIT project aims at achieving such a solution as explained in those three topics by enabling a more frequent and dynamic interaction between ISPs and CPs in order to harmonize objectives of each stakeholder and reach, through collaboration, mutually benefiting operation levels. At the same time the incorporation of information from SNPs and QoE feedback from end users will lead to new business relationships between stakeholders. SmartenIT will provide a framework allowing intermediary ISPs to reach specific agreements so as to fulfill requirements of CPs.

Clouds. Clouds (special case of overlay networks) emerged as a major paradigm for the current and the Future Internet [7, 8]: First, CPs see a strong decentralization, such in multiple coordinated data centers, spread across different continents of the world [9]. In turn, different management scopes for CPs with multiple Points-of-Presence (PoP), the distributed DC's cloud infrastructure itself, and the internal management coordination determine the key for an operational and commercial success [10]. Second, CPs largely benefit from economies of scale, since resources, such as storage or CPU power, can be shared among a large number of users. This makes CPs more energy-efficient and less expensive than traditional applications [11, 12]. Third, it simplifies administrative and maintenance tasks (software updates and backups) considerably. Fourth, it permits users to access easily their applications and large amounts of private or public data from different locations and devices.

As a consequence, a large variety of overlay applications (i.e. applications served by and/or spanning resources that are scattered in the cloud) already exist, e.g., online office applications like Google documents, data repositories such as dropbox.com, or cloud gaming as offered by OnLive.com. Secondly, the support of those in the wired and wireless access network domain is a commercial must, as end-users expect that.

Today, cloud applications are very popular since they can be accessed from all over the world with different devices. However, cloud and network management methods are limited to technical parameters such as network load or CPU utilization. These criteria are of importance for CPs and ISPs, but have - if at all - a limited importance to end-users. End-users perceive the quality of cloud applications in a different manner. For them, responsiveness and usability are key parameters that decide 
which applications users like. Cloud computing offers different services and many overlay applications run on top of clouds, where energy-related costs are becoming one of the largest contributors to the overall cost of operating a DC, whereas the degree of DC utilization continues to be very low.

Social Networks. Another important case of overlay networks are Online Social Networks (OSN). In particular, OSNs such as Facebook or Twitter, are today a pervasive part of the Internet and aim to cover the entire digital live of its users in the near future $[13,14,15]$. On one hand, they can be considered as one type of cloud applications, where users organize their social relations, chat, or post their current activities or mood. On the other hand, they deserve high emphasis, since they contain information about user relations, interests, and the popularity of contents available in the Internet, such as movies or songs. Moreover, a significant part of requests for content is placed over SNPs. Facebook remains since 2009 the 3rd largest online video content provider in the US. Hence, based on social relations between Facebook users, one can deduce important observations that have to do with the dissemination patterns of such content in these environments. The structure of OSNs will be exploited for traffic management strategies, like caching or pre-fetching, together with additional useful information stemming from OSNs, e.g., on popularity.

SmartenIT does not only consider OSNs, they will also be used as a source of information about the popularity of specific other cloud applications or content. In this way, OSNs connect user behavior and user expectations to cloud and network management methods. For example, applications that many persons of a specific geographical region in an OSN suggest to their friends will get popular in this area. As a result, allocating cloud storage and processing power in DCs in this region of the world will improve the QoE perceived by users. Thus, SmartenIT will develop interaction mechanisms and paradigms between OSN information and cloud and network management decisions. This tight interaction between management decisions and the information contained in OSNs has not been studied in depth so far, leading to an effective exploitation for traffic management purposes.

\section{Challenges Emerging from Internet Trends}

These trends in the application domain pose several major challenges for the current structure of the Internet, from a technical and economic point of view [4]:

1st Challenge: Emerging overlay applications, such as cloud applications or OSNs, make traffic management difficult for ISPs, since many of them run on different DCs and ISPs have no influence to which DC a specific user connects to or on which server the user will be served by. Additionally, large ISPs establish their own network management for the transport of all best effort type traffic within their platform under cost optimization constraints. A lack of coordination between ISPs and overlay providers detracts from globally optimized transport paths [16]. Traffic generated by cloud applications will have unpredictable patterns, crossing many and different 
domains, due to the mobility of cloud users or the distribution of cloud resources. Moreover, the traffic of certain cloud applications may be cacheable, often by different stakeholders, while other traffic is not. Although caching in distributed DCs, in broadband access network nodes, and on user devices improves application performance in many scenarios, the interoperability of caching mechanisms leaves many open issues [6, 16]. Thus, SmartenIT will trade-off between cache-ability and acceptable degradation of performance and QoE, depending on the application [17]. Moreover, SmartenIT will investigate how the coordination can be improved across administration boundaries with different incentives for each involved party.

2nd Challenge: The usability of overlay applications strongly depends on network conditions and Quality-of-Service (QoS) guarantees on the path between the DC and the end-user, which might be controlled by several different ISPs [18]. Furthermore, end-users judge the usability of an application based on their own experience, which is referred to as the QoE. Hence, SmartenIT manages overlay application needs in consideration of crucial QoE. That means that management approaches have to (1) be able to measure or at least estimate the QoE that overlay applications deliver to the end user and (2) use this metric as an important parameter to optimize for. The QoE metric is based on measurable parameters within the network (e.g., packet loss) or at the application layer (e.g., number of stalling events for HTTP-based video streaming). Hence, the QoE metric allows for monitoring the network or the application and later on for evaluating mechanisms developed in SmartenIT with respect to QoE.

3rd Challenge: The variety of often proprietary overlay applications makes it difficult to combine services from different overlay providers to a new service; an example is cloud computing [8, 19]. This implies that application developers need the flexibility to choose already implemented services from different clouds and combine them to provide a new service. Open protocols contain a potential to make the cloud paradigm more powerful $[4,20,21]$, however, they are not widely adopted. Thus, SmartenIT addresses composed services from different clouds with new traffic management mechanisms to be influenced by economic criteria and standard requirements, e.g., bandwidth or QoE. It will be important to choose and study overlay applications, which simultaneously have an important impact on network evolution and management, terminals, and user behavior. Such potentially influential applications are, e.g., YouTube, Dropbox, or Facebook. Another viewpoint is related to the commercial market of IPTV applications with Video-on-Demand additions, which are offered by telecommunication service providers and ISPs.

4th Challenge: The trade-off between a cloud computing computational efficiency and the energy consumption also arises with respect to traffic implications. This has to be addressed by a cross-layer management approach [22]. Since users use OSNs to share information on the content they are interested in, the demand for a high-quality content may grow fast. Hence, it is crucial for an innovative CDN to be sociallyaware, predict the demand, and avoid resource exhaustion and QoE degradation [23]. However, this may come at a cost due to higher computational complexity or energy consumption. Therefore, SmartenIT applies an energy-efficient traffic management in 
the context of overlay applications. QoE needs be assessed on a per application basis, since the user-perceived quality of an application depends strongly on the type of application. As a consequence, SmartenIT will define QoE indicators for cloud applications and to derive their power to predict user satisfaction.

5th Challenge: The fact that mobile cloud devices are limited in their battery lifetime and by mobile data caps imposes strict requirements on respective management mechanisms, while considering mobile device's limited battery resources. The energyefficient access of cloud-based services over Wifi-based ad-hoc networks shows a high potential to reduce traffic in cellular networks, while increasing the download throughput at the same time as reducing the energy consumption despite additional overhead. To be able to estimate energy consumption of different overlay approaches for management of mobile cloud applications, SmartenIT verifies accurate models for the energy consumption of mobile devices, which can simulate overhead introduced by different management approaches.

6th Challenge: Stakeholders of today's and tomorrow's Internet with conflicting interests make decisions that may lead the system into a suboptimal situation. Therefore, traffic management approaches need to provide incentives to stakeholders to support mutually beneficial decisions. Relevant mechanisms address incentives translating either directly or indirectly to an economic benefit arising by the improvement of an index (such as the level of locality or QoS). Therefore, SmartenIT designs solutions that employ appropriate incentive mechanisms to constitute the deployment and use of overlay applications more efficiently.

\section{Use Cases and Solution Approaches}

For demonstrating how to tackle those emerging challenges, SmartenIT follows a use case driven approach. Selected scenarios show the key potential of the SmartenIT approach for the quality of applications for residential and commercial users, for business opportunities for both ISPs and telecommunication service providers, and for value-added service providers. The final decision on the most important scenario(s) will be based on basic evaluations and a detailed requirements analysis.

Inter-cloud Communication: Three classes of services are offered by clouds: (1) storage, (2) CPU and RAM, and (3) applications. Additionally, an integration of components of (1) and (2) provided by different clouds is possible. The exchange of data between storage and computational resources is performed by the network. Clouds physically connected to different network operators require a multi-domain operation topology and applications may demand for a guaranteed QoS level. Therefore, the exchange of data between storage and computational resources is performed by the network. While setting the connection, QoS/QoE should be taken into account as well as the connection cost, which is depending on the selected route. Some services require an ongoing dynamic allocation of resources due to the migration of virtual machines within the cloud from one physical location to another. Changing locations 
may require a new connection to be established between new cloud servers, regardless of whether they belong to the same $\mathrm{CP}$ or to inter-connected ones. An algorithm for the selection of a new physical location of services or resources in the cloud should take into account the following criteria: (a) data transfer costs, (b) QoS/QoE assurance, (c) service (e.g., MPLS, L2VPN, or L3VPN) availability, (d) service setup costs, (e) network resource availability, and (f) data transfer security. The migration of a service to the cloud offering the same type of resources, but being managed by a distinct provider can be addressed by such an algorithm. This scenario is particularly relevant for the many emerging Virtual DC (VDC) products recently pushed into the market by many network operators, such as Interoute's VDC. In particular, these VDC solutions are attractive for enterprise customers willing to move network services to the (private) cloud. They are becoming an impelling requirement to support services on top of a multi-tenant cloud infrastructure, especially for large enterprise users with many remote offices.

Exploiting OSN Information - Social Awareness: Recently OSNs have become very popular. Facebook currently claims 845 million monthly active users each month, with 250 million photos uploaded daily and 450 million mobile users. User activities in OSNs such as micro-blogging (Twitter) and exchange of photos and videos (Facebook) are responsible for huge portions of the overall Internet traffic [1]. This overlay traffic generated by OSNs implies congestion increase within ISPs and an increase of operating costs, especially for inter-domain traffic. This is in particular the case for video traffic that is induced by OSNs, e.g., if a user posts a link to a video served by a CDN and recommends this video to his friends within the OSN. As a consequence, the structure of OSNs and the information diffusion in OSNs have to be analyzed. Thus, related work and existing measurement data, e.g., for Facebook or Twitter, have to be analyzed in terms of OSN structure and information diffusion. Though a major attribute that characterizes OSNs is the existence of a many metainformation regarding social relationships among their users, e.g., family members and friends (Facebook), colleagues and other professionals (LinkedIn), or people are interested in similar hobbies/activities (MySpace for music bands, songs and video clips, Flickr for photography lovers). The exploitation of such meta-information by ISPs can lead to socially-aware traffic management mechanisms that aim at (1) more sophisticated traffic management, (2) the reduction of traffic load on inter-domain links, and (3) a reduction of operating costs for ISPs. For instance, a video could be cached (pre-fetched) near "friends" of an OSN user, who downloads videos that this user shares with them, while a video containing an interesting lecture could be cached near the users' colleagues. The caching of a video near groups of users that would be interested in it, would allow for an operating cost minimization (avoiding redundant downloads from remote locations). In SmartenIT, the question will be addressed to discover what meta-information can be used, under which circumstances can this information be acquired (e.g., special agreements of OSN providers and the ISPs, incentives of stakeholders), and how to employ traffic management more efficiently for all parties. 


\section{$5 \quad$ Requirements for System Design and Architecture Design}

The concrete use cases considered in SmartenIT will pose requirements for the system design. In particular, the initial set of requirements, partially discussed above with respect to the approaches for such use cases, will be applied to derive a respective set of architectural components, functions, and interactions, which can generalize those use cases' claims. SmartenIT's preliminary architecture outlines the key properties and functionality of the SmartenIT's approach, each of which span one or multiple domains out of the overlay/cloud domain, the core network domain, and the user/access network domain. Those key properties of SmartenIT's architecture include the three main SmartenIT objectives QoE-/social-awareness, energy efficiency, and incentive-compatibility/ economic efficiency, all of which are equally relevant in all three domains. Key functionality of the overlay/cloud domain (with overlap to the core network domain) includes the overlay management and the cloud traffic management. Likewise, key functionality rooted in the core network domain (with overlap to the overlay/cloud domain) include $\mathrm{CD} /$ caching, network traffic management potentially supported by a Software-defined Networks (SDN)-based approach - and traffic monitoring. Finally, key functionality in the user/access network domain includes the QoS/QoE and mobile traffic management.

QoE-Awareness. The SmartenIT architecture will rely on QoE as one of the key parameters for network and cloud management. This will shift the focus from technical parameters toward user expectations. Mapping between QoS network parameters and the user-perceived service quality, namely QoE, has received a lot of attention in recent years [24]. Development of QoS/QoE models and functions mapping QoS parameters (e.g., packet loss, delay, or jitter) into QoE metrics is promising, which may support the provisioning of services over heterogeneous networks with high and stable QoE. It is, however, not easy, since QoS/QoE mapping models are strongly dependent on application/service types and technical as well as non-technical aspects [25]. SmartenIT will examine QoS/QoE relations for cloud-based service and selected SmartenIT scenarios and applications. Further, ongoing activities in standardization bodies will be followed and utilized, like the proposal for a framework for an opinion model for web-browsing QoE in the ITU-T work group SG12 on "Performance, QoS and QoE", which may be the basis for QoS/QoE mappings and QoE monitoring.

Social-Awareness. Due to the fast propagation of information via OSNs, a growth of content popularity (and application reputation) may occur. E.g., people sharing links to content may cause a rapid increase of demands in some area of the network and a very high load in DCs and networks. As a result, it may be difficult for an ISP to serve all demands efficiently and users may not receive high QoE. Relying on both social- and QoE-awareness, it will become possible to predict such situations and, by proactive reactions, an ISP will be prepared to rapid growth of demands, e.g., by content, server, application replication or reorganization of resources in the cloud.

Economic Efficiency. The shift of computation and data into the cloud has become a key trend in the Internet. With the market approaching perfect competition, the 
perceived service quality will become an important differentiator between CPs, as the customer is able to choose between different competing providers. For this reason, understanding and managing QoE of end-users provides huge opportunities for CPs to put themselves at an advantage. It enables CPs to observe and react quickly to quality problems, at best before customers perceive them and start churning. From an economic perspective, an optimum QoE has to be achieved while constraining the application to behave as efficient as possible in order to minimize operational costs.

As several QoE evaluation methods exist, they can be classified as subjective tests, objective evaluation, or models. The category of subjective tests encompasses a wide group of methods that involve real users as evaluators. They range from laboratory tests (well prepared experiments in a controlled environment and precisely prepared test scenarios) through crowd-sourcing [26] to test-bed experiments to ask customers a set of questions after using the service (e.g., Skype). A QoE evaluation metric is also the user churn that may be assessed statistically. A second group of QoE evaluation methods is the objective evaluation. These methods do not involve real users. User-perceived quality is approximated on the basis of an examination of selected features of delivered service itself (non-reference methods) or by comparing the source quality to the one received by a customer (full/reduced reference methods).

Finally, QoE models enable the assessment of QoE without involving real users but via examining network conditions (e.g., QoS parameters) and other factors including user device capabilities. Usually such models are not general, but they are closely related to a given service and are tightly connected with its characteristics. They are developed with a support of subjective tests. Once a model, e.g., QoS-QoE, mapping is determined, the QoE may be assessed without involving humans. The advantage of this approach is also that there is no need to examine directly the received content quality itself. For example, [27] proposes a QoE model for YouTube video streaming. With this model, a service provider can monitor QoS parameters (like throughput) and react in advance if their values approach the level that may result in QoE degradation.

Energy Efficiency. The SmartenIT architecture will address the energy-aware dynamic provision of resources based on the consolidation of existing application, while simultaneously addressing under-utilization of servers and in turn reducing energy costs. Thus, energy costs cannot be treated separately from resource provision and allocation. [28] models the problem of minimizing energy consumption of the allocation of resources to networked applications as a Stackelberg leadership game to find an upper bound of energy saving. This model is applied in SmartenIT to a proportional-share mechanism, where resource providers maximize their profit by minimizing energy costs, while users select resources ensuring minimum requirements are satisfied. This mechanism can determine the optimal set of resources, even in realistic conditions considering incomplete information and heterogeneous applications.

\section{Summary and Preliminary Conclusions}

From the perspective of a multi-stakeholder viewpoint and highly unregulated services market within the Future Internet the management of traffic by respective network 
and service management mechanisms determines the crucial interface between user demands and service perceptions, networking technology, and operational costs. Thus, SmartenIT's contributions in the clear preparations of the starting point for such investigations indicated that at least two major scenarios will drive the stakeholders' requirements analysis, including conflicting interests, cooperation possibilities, and incentives. This overview has outlined in detail the technical and partially economic basis for a list of optimizations to be considered.

Especially from the perspective of ISPs, APs, and CPs the aim of QoE-awareness is offering a high and stable level of perceived quality to prevent customers' churn and also to increase attractiveness of that offer within overlay or cloud services. Since a high and stable QoE is currently considered crucial for marketability of applications and services, QoE control is desired to enable an advance reaction to imminent quality degradation that would result in user dissatisfaction.

However, many management approaches toward OSNs, overlay service, and QoE management are mainly designed for only a single domain of a single stakeholder. Therefore, their effectiveness suffers from an inherent lack of information exchange between all involved constituents, including service infrastructure (IaaS, PaaS, SaaS), ISPs and telecommunication service providers, and end-users (private and business). To remedy this key problem SmartenIT works on flexible cooperation between stakeholders involved, ultimately enabling every user to (a) access the offered cloud service in any contexts and (b) share content, interact, and collaborate with other users in a dynamic, seamless, and transparent way while maximizing QoE at the same time. As such SmartenIT started the development of QoE-aware network and resource management mechanisms to enable dynamic and proactive QoE support for cloudbased services. Appropriate QoE evaluation methodology and tools will be selected, developed, improved, and used at various stages of SmartenIT solutions development.

Acknowledgements. This work has been performed in the framework of the EU ICT STREP SmartenIT (FP7-ICT-2011-317846). The authors would like to thank the entire SmartenIT team for their discussions and input on major research problems.

Open Access. This article is distributed under the terms of the Creative Commons Attribution Noncommercial License which permits any noncommercial use, distribution, and reproduction in any medium, provided the original author(s) and source are credited.

\section{References}

[1] Cisco: Cisco Global Cloud Index: Forecast and Methodology, 2010-2015, White Paper (2011)

[2] Cisco: Hyper-connectivity and the Approaching Zetabyte Era (2010)

[3] Cisco: Cisco Visual Networking Index: Global Mobile Data Traffic Forecast Update 2010-2015, White Paper (2011)

[4] Stankiewicz, R., Jajszczyk, A.: A Survey of QoE Assurance in Converged Networks. Computer Networks 55(7), 1459-1473 (2011) 
[5] Hoßfeld, T., et al.: An Economic Traffic Management Approach to Enable the TripleWin for Users, ISPs, and Overlay Providers. In: Tselentis, G., et al. (eds.) FIA Prague Book "Towards the Future Internet - A European Research Perspective". IOS Press Books Online (2009)

[6] IETF: Content Delivery Networks Interconnection (Active WG), http: //tools.ietf.org/wg/cdni/ (last visited February 2013)

[7] Cisco: Cisco Cloud Computing - Data Center Strategy, Architecture and Solutions (2009)

[8] Papazoglou, M.P., van den Heuvel, W.: Blueprinting the Cloud. IEEE Internet Computing 15(6), 74-79 (2011)

[9] Cisco: Cisco Secure Network Container: Multi-Tenant Cloud Computing (2010)

[10] Poese, I., Frank, B., Ager, B., Smaragdakis, G., Feldmann, A.: Improving Content Delivery Using Provider-aided Distance Information. In: 10th Annual Conference on Internet Measurement (IMC), Melbourne, Australia (2010)

[11] Chen, G., et al.: Energy-aware Server Provisioning and Load Dispatching for Connectino-intensive Internet Services. In: 5th USENIX Symposium on Networked Systems Design and Implementation, San Francisco, Calfornia, U.S.A. (2008)

[12] Zhang, Q., Cheng, L., Boutaba, R.: Cloud Computing: State-of-the-art and Research Challenges. Journal of Internet Services and Applications 1(1), 7-18 (2010)

[13] Cha, M., Haddadi, H., Benevenuto, F., Gummadi, K.P.: Measuring User Influence in Twitter: The Million Follower Fallacy. In: 4th International AAAI Conference on Weblogs and Social Media (ICWSM), Washington, D.C., U.S.A. (2010)

[14] Krishnamurthy, B.: A Measure of Online Social Networks. In: 1st International Workshop on Communication Systems and Networks and Workshops (COMSNETS 2009), Bangalore, India (2009)

[15] Gjoka, M., Sirivianos, M., Markopoulou, A., Yang, X.: Poking Facebook: Characterization of OSN Applications. In: 1st Workshop on Online Social Networks (WOSN), Seattle, Washington, U.S.A. (2008)

[16] Haßlinger, G., Hartleb, F.: Content Delivery and Caching from a Network Provider's Perspective. Computer Networks 55(18), 3991-4006 (2011)

[17] Chiu, D., Shetty, A., Agrawal, G.: Elastic Cloud Caches for Accelerating ServiceOriented Computations. In: ACM/IEEE International Conference for High Performance Computing, Networking, Storage and Analysis, New Orleans, Louisiana, U.S.A. (2010)

[18] Hoßfeld, T., Fiedler, M., Zinner, T.: The QoE Provisioning-Delivery-Hysteresis and Its Importance for Service Provisioning in the Future Internet. In: 7th Conference on Next Generation Internet Networks (NGI), Kaiserslautern, Germany (2011)

[19] Bernstein, D., Ludvigson, E., Sankar, K., Diamond, S., Morrow, M.: Blueprint for the Intercloud - Protocols and Formats for Cloud Computing Interoperability. In: 4th International Conference on Internet and Web Applications and Services (ICIW), Venice/Mestre, Italy (2009)

[20] Chieu, T.C., Mohindra, A., Karve, A., Segal, A.: Solution-based Deployment of Complex Application Services on a Cloud. In: IEEE International Conference on Service Operations and Logistics and Informatics (SOLI), Qingdao, China (2010) 
[21] Konstantinou, A.V., et al.: An Architecture for Virtual Solution Composition and Deployment in Infrastructure Clouds. In: 3rd International Workshop on Virtualization Technologies in Distributed Computing (VTDC), Barcelona, Spain (2009)

[22] Schulz, G.: Cloud and Virtual Data Storage Networking. Auerbach Publications (2011)

[23] Scellato, S., Mascolo, C., Musolesi, M., Crowcroft, J.: Track Globally, Deliver Locally: Improving Content Delivery Networks by Tracking Geographic Social Cascades. In: 20th International Conference on World Wide Web (WWW), Hyderabad, India (2011)

[24] Fiedler, M., Hoßfeld, T., Tran-Gia, P.: A Generic Quantitative Relationship between Quality of Experience and Quality of Service. IEEE Network 24(2), 36-41 (2010)

[25] Hoßfeld, T., Schatz, R., Varela, M., Timmerer, C.: Challenges of QoE Management for Cloud Applications. IEEE Communications Magazine 50(4), 28-36 (2012)

[26] Hoßfeld, T., et al.: Quantification of YouTube QoE via Crowdsourcing. In: IEEE International Workshop on Multimedia Quality of Experience - Modeling, Evaluation, and Directions (MQoE), Dana Point, California, U.S.A. (2011)

[27] Hoßfeld, T., Schatz, R., Biersack, E., Plissonneau, L.: Internet video delivery in youTube: From traffic measurements to quality of experience. In: Biersack, E., Callegari, C., Matijasevic, M. (eds.) Data Traffic Monitoring and Analysis. LNCS, vol. 7754, pp. 264-301. Springer, Heidelberg (2013)

[28] Leon, X., Navarro, L.: Limits of Energy Saving for the Allocation of Data Center Resources to Networked Applications. In: IEEE INFOCOM 2011, Shanghai, China (2011) 